\title{
PLURISUBHARMONIC FUNCTIONS OUTSIDE COMPACT SETS URBAN CEGRELL
}

(Communicated by Irwin Kra)

\begin{abstract}
We study the Monge-Ampère mass of plurisubharmonic functions defined outside a compact set.
\end{abstract}

1. Introduction. Let $\Omega$ be an open and bounded pseudoconvex set in $\mathbf{C}^{n}$, $n \geq 2$, and $K$ a compact subset of $\Omega$ so that $\Omega \backslash K$ is connected. By the Hartogs extension theorem, every analytic function on $\Omega \backslash K$ extends to $\Omega$. When it comes to plurisubharmonic functions the situation is different.

The purpose of this note is to study the Monge-Ampère mass $\int_{\Omega^{\prime} \backslash K}\left(d d^{c} u\right)^{n}$, where $u \in \mathrm{PSH} \cap L_{\text {loc }}^{\infty}(\Omega \backslash K)$ and $K \Subset \Omega^{\prime} \Subset \Omega$.

The case when $u \in \mathrm{PSH} \cap C^{\infty}(\Omega \backslash K)$ has already been studied by Fornaess and Sibony [3] and Griffith [4].

\section{Estimates.}

Proposition. Assume that $\varphi \in \mathrm{PSH} \cap L_{\mathrm{loc}}^{\infty}(\Omega \backslash K)$, where $K$ is a removable singularity set for the plurisubharmonic functions (cf. [2]). Then $\int_{\Omega^{\prime} \backslash K}\left(d d^{c} \varphi\right)^{n}<$ $+\infty$.

ProOF. By Bedford and Taylor $[\mathbf{1}],\left(d d^{c} \varphi\right)^{n}$ is a well-defined positive measure on $\Omega \backslash K$. Since $\varphi$ extends to a plurisubharmonic function on $\Omega$, we can choose a sequence $\varphi_{j} \in \mathrm{PSH} \cap C^{\infty}$ near $\overline{\Omega^{\prime}}$ such that $\varphi_{j} \searrow \varphi, j \rightarrow+\infty$.

Let $W=\left\{z \in \Omega^{\prime}, d(z, K)>\frac{1}{2} d\left(\partial \Omega^{\prime}, K\right)\right\}$. Then $\delta=\inf _{z \in W} \varphi(z)>-\infty$, and if we put $\psi_{j}=\sup \left(\varphi_{j}, \delta\right)$, then $\psi_{j}=\varphi_{j}$ on $W$. So $\int_{\Omega^{\prime}}\left(d d^{c} \psi_{j}\right)^{n}=\int_{\Omega^{\prime}}\left(d d^{c} \varphi_{j}\right)^{n}$ by Stokes' theorem. Therefore,

$$
\begin{aligned}
\int_{\Omega^{\prime} \backslash K}\left(d d^{c} \varphi\right)^{n} & \leq \lim _{j \rightarrow+\infty} \int_{\Omega^{\prime}}\left(d d^{c} \varphi_{j}\right) \\
& =\lim _{j \rightarrow+\infty} \int_{\Omega^{\prime}}\left(d d^{c} \psi_{j}\right)^{n} \\
& =\int_{\Omega^{\prime}}\left(d d^{c} \sup (\varphi, \delta)\right)^{n}<+\infty
\end{aligned}
$$

since $\sup (\varphi, \delta) \in \mathrm{PSH} \cap L_{\text {loc }}^{\infty}(\Omega)$.

The next theorem is a generalization of Theorem 2.2 in Fornaess and Sibony [3].

THEOREM. Let $\psi \in \mathrm{PSH} \cap C^{2}(\Omega)$ and assume that $\Omega^{\prime}=\{\psi<1\}$ and that $K=\{\psi \leq s\}$ for an $s, 0<s<1$. If $\varphi \in \operatorname{PSH} \cap L_{\text {loc }}^{\infty}(\Omega \backslash K)$, then

$$
\int_{\{s<\psi<1\}}|\varphi|\left(d d^{c} \varphi\right)^{n-1} \wedge d d^{c} \psi<+\infty
$$

Received by the editors January 22, 1987.

1980 Mathematics Subject Classification (1985 Revision). Primary 32F05; Secondary 31C10. 
and

$$
\int_{\{s<\psi<1\}}(\psi-s)\left(d d^{c} \varphi\right)^{n}<+\infty .
$$

PrOOF. Since $\Omega$ is pseudoconvex and $\psi$ plurisubharmonic, there is, to every $z_{0} \in\{s<\psi<1\}$, an analytic function such that $f(z) \neq f\left(z_{0}\right) \forall z \in K$. If we restrict $\varphi$ to $\left\{f(z)=f\left(z_{0}\right)\right\}$ and apply the maximum principle, we conclude that $\varphi$ is uniformly bounded above on $\overline{\Omega^{\prime} \backslash K}$. It is therefore no restriction to assume that $\varphi \leq 0$.

Let $s<r<1$. Then, by Stokes' theorem we have

$$
\begin{aligned}
& \int_{\{r<\psi<1\}}-\varphi\left(d d^{c} \psi\right) \wedge\left(d d^{c} \varphi\right)^{n-1}+\int_{\{r<\psi<1\}}(\psi-r)\left(d d^{c} \varphi\right)^{n} \\
& \leq \frac{\lim }{t \searrow r} \int_{\{r<\psi<1\}}-\varphi d d^{c}(\max \psi, t) \wedge\left(d d^{c} \varphi\right)^{n-1}+\int_{\{r<\psi<1\}}(\psi-r)\left(d d^{c} \varphi\right)^{n} \\
& =\frac{\lim }{t \searrow r}\left[\int_{\{\psi=1\}}-\varphi d^{c}(\max \psi, t) \wedge\left(d d^{c} \varphi\right)^{n-1}\right. \\
& \left.\quad+\int_{\{r<\psi<1\}} d \max (\psi, t) \wedge d^{c} \varphi \wedge\left(d d^{c} \varphi\right)^{n-1}+\int_{\{r<\psi<1\}}(\psi-r)\left(d d^{c} \varphi\right)^{n}\right] \\
& =\frac{\lim }{t \searrow r}\left[\int_{\{\psi=1\}}-\varphi d^{c}(\psi, t) \wedge\left(d d^{c} \varphi\right)^{n-1}\right. \\
& \quad+\int_{\{\psi=1\}}(\max (\psi, t)-r) d^{c} \varphi \wedge\left(d d^{c} \varphi\right)^{n-1} \\
& \quad-\int_{\{\psi=r\}}(\max (\psi, t)-r) d^{c} \varphi \wedge\left(d d^{c} \varphi\right)^{n-1} \\
& =\int_{\{\psi=1\}}-\varphi d^{c} \psi \wedge\left(d d^{c} \varphi\right)^{n-1}+\int_{\{\psi=1\}}(\psi-r) d^{c} \varphi \wedge\left(d d^{c} \varphi\right)^{n-1} .
\end{aligned}
$$

Since the right-hand side is uniformly bounded in $r, s<r<1$, and since $\varphi \leq 0$, we get

$$
\int_{\{s<\psi<1\}}|\varphi| d d^{c} \psi \wedge\left(d d^{c} \varphi\right)^{n-1}+\int_{\{s<\psi<1\}}(\psi-s)\left(d d^{c} \varphi\right)^{n}<+\infty,
$$

where each member is nonnegative.

3. An example. The following example shows that the convergence factor $\psi-s$ really is needed in the Theorem. There are functions $\varphi$ and $\psi$ such that $\int_{\{s<\psi<1\}}\left(d d^{c} \varphi\right)^{n}=+\infty$, where $\varphi$ can be taken to be plurisubharmonic and bounded.

Define $u(z)=-\sqrt{|z|^{2}-\frac{1}{2}} ;$ then $u$ is subharmonic on $\left\{z \in \mathbf{C} ; \frac{1}{2}<|z|^{2}<1\right\}$, $-1 / \sqrt{2}<u<0$, and $\Delta u=\left(1-|z|^{2}\right) /\left(|z|^{2}-\frac{1}{2}\right)^{3 / 2}$. 
Define $\psi(z, w)=|z|^{2}+\frac{2}{3}|w|^{8}$, and let $0<\eta<\frac{1}{20}$, and put

$\varphi_{\eta}(z, w)=\max \left[-\frac{1}{2} \sqrt{(1+\eta)^{2}|z|^{2}-\frac{1}{2}+|w|^{2}},|w|^{2}+(1+\eta)|w|^{4}-\sqrt{\frac{3}{2}\left(\eta+\eta^{2} / 2\right)}\right]$.

Then $\varphi_{\eta}$ is plurisubharmonic on $\left\{\frac{1}{2}<\psi<(20 / 21)^{2}\right\}$ for, if $(1+\eta)^{2}|z|^{2} \leq \frac{1}{2}$, we have, when $\psi(z, w)>\frac{1}{2}$,

$$
\begin{aligned}
& \frac{2}{3}|w|^{8}>\frac{1}{2}-|z|^{2}>\frac{1}{2}\left(1-(1+\eta)^{-2}\right)=\frac{1}{2}\left(2 \eta+\eta^{2}\right) /(1+\eta)^{2}=\left(\eta+\eta^{2} / 2\right)(1+\eta)^{2} \\
& \text { so } \\
& \qquad(1+\eta)|w|^{4}>\sqrt{\frac{3}{2}\left(\eta+\eta^{2} / 2\right)} .
\end{aligned}
$$

Assume now that $\frac{1}{2}<|z|^{2}+\frac{2}{3}|w|^{8}<(20 / 21)^{2}$ and that

Then

$$
\sqrt{\frac{3}{5}} \frac{\sqrt{\eta+\eta^{2} / 2}}{1+\eta}<|w|^{4}<\sqrt{\frac{2}{3}} \frac{39}{40} \frac{\sqrt{\eta+\eta^{2} / 2}}{1+\eta}
$$

if

$$
\varphi_{\eta}=-\frac{1}{2} \sqrt{(1+\eta)^{2}|z|^{2}-\frac{1}{2}}+|w|^{2}
$$

$$
\begin{gathered}
-\frac{1}{2} \sqrt{(1+\eta)^{2}|z|^{2}-\frac{1}{2}}>(1+\eta)|w|^{4}-\sqrt{\frac{3}{2}\left(\eta+\eta^{2} / 2\right)} \\
\Leftrightarrow(1+\eta)^{2}|z|^{2}<\frac{1}{2}+4\left(\sqrt{\frac{3}{2}\left(\eta+\eta^{2} / 2\right)}-(1+\eta)|w|^{4}\right)^{2}
\end{gathered}
$$

which holds if

$$
\left(\frac{1}{2}-\frac{2}{3}|w|^{8}\right)(1+\eta)^{2}<(1+\eta)^{2}|z|^{2}<\frac{1}{2}+4\left(\sqrt{\frac{3}{2}\left(\eta+\eta^{2} / 2\right)}-(1+\eta)|w|^{4}\right)^{2}
$$

which is a well-defined domain if

$$
\begin{aligned}
\eta+\eta^{2} / 2 & -\frac{2}{3}|w|^{8}(1+\eta)^{2} \\
& <6\left(\eta+\eta^{2} / 2\right)-8 \sqrt{\frac{3}{2}\left(\eta+\eta^{2} / 2\right)}(1+\eta)|w|^{4}+4(1+\eta)^{2}|w|^{8} \\
& \Leftrightarrow 0<5\left(\eta+\eta^{2} / 2\right)+\frac{14}{3}(1+\eta)^{2}|w|^{8}-8 \sqrt{\frac{3}{2}} \sqrt{\eta+\eta^{2} / 2}(1+\eta)|w|^{4}
\end{aligned}
$$

which holds with $|w|^{4}$ in the interval above-the right-hand side is then strictly larger than

$$
5\left(\eta+\frac{\eta^{2}}{2}\right)+\frac{14}{3}(1+\eta)^{2} \frac{3}{5} \frac{\eta+\eta^{2} / 2}{(1+\eta)^{2}}-8 \cdot \frac{39}{40}\left(\eta+\frac{\eta^{2}}{2}\right)=0
$$

Therefore,

$$
\int_{\frac{1}{2}<|z|^{2}+\frac{2}{3}|w|^{8}<(20 / 21)^{2}}\left(d d^{c} \varphi_{\eta}\right)^{2} \geq c \int_{A_{\eta}} \frac{d z d w}{\left((1+\eta)^{2}|z|^{2}-\frac{1}{2}\right)^{3 / 2}}
$$

where

$$
A_{\eta}=\left\{\begin{array}{l}
\left(\frac{1}{2}-\frac{2}{3}|w|^{8}\right)(1+\eta)^{2}<(1+\eta)^{2}|z|^{2}<\frac{1}{2}+4\left(\sqrt{\frac{3}{2}\left(\eta+\eta^{2} / 2\right)}-(1+\eta)|w|^{4}\right)^{2} \\
\frac{\sqrt{\eta+\eta^{2} / 2}}{1+\eta} \alpha<|w|^{4}<\beta \frac{\sqrt{\eta+\eta^{2} / 2}}{1+\eta}
\end{array}\right.
$$


and where we have chosen $\sqrt{\frac{3}{5}}<\alpha<\beta<\frac{39}{40} \sqrt{\frac{2}{3}}$ and where $c$ is a strictly positive constant. The right-hand side is not smaller than

$$
\begin{aligned}
c\left\{\left(\alpha^{2}-\frac{3}{5}\right)+\frac{39}{40} \sqrt{\left.\frac{2}{3}-\beta\right\}}\right. & \int \frac{\left(\eta+\eta^{2} / 2\right) d w}{2^{3}\left(\sqrt{\frac{3}{2}\left(\eta+\eta^{2} / 2\right)}-(1+\eta)|w|^{4}\right)^{3}} \\
\frac{\sqrt{\frac{3}{5}\left(\eta+\eta^{2} / 2\right)}}{1+\eta} & <|w|^{4}<\sqrt{\frac{2}{3} \frac{39}{40} \frac{\sqrt{\eta+\eta^{2} / 2}}{1+\eta}} \\
& \geq d\left(\eta+\eta^{2} / 2\right)^{1-3 / 2+1 / 4} \\
& \left.=\frac{d}{\left(\eta+\eta^{2} / 2\right)^{1 / 4}} \rightarrow+\infty, \quad \eta \rightarrow 0 \text { (d pos. const. }\right) .
\end{aligned}
$$

Furthermore, $-\frac{1}{2}<\varphi_{\eta} \leq 2$ so if we put

$$
\varphi=\sum_{j=20}^{\infty} \frac{1}{j^{2}} \varphi_{1 / j^{4}}
$$

then

$$
\varphi \in \mathrm{PSH} \cap L^{\infty}\left(\frac{1}{2}<|z|^{2}+\frac{2}{3}|w|^{8}<\left(20^{4} /\left(20^{4}+1\right)\right)^{2}\right)
$$

and

$$
\int_{\frac{1}{2}<|z|^{2}+\frac{2}{3}|w|^{8}<(20 / 21)^{2}}\left(d d^{c} \varphi\right)^{2}=+\infty .
$$

REMARK. It is possible to modify the $\varphi_{\eta}$ 's to get the function $\varphi C^{\infty}$ on $\Omega \backslash\{w=0\}$. We do not know if one can modify $\varphi$ to be $C^{\infty}$ on $\Omega$ but still have $\int_{\Omega \backslash K}\left(d d^{c} \varphi\right)^{2}=+\infty$.

\section{REFERENCES}

1. E. Bedford and B. A. Taylor, A new capacity for plurisubharmonic functions, Acta Math. 199 (1982), 1-40.

2. U. Cegrell, Removable singularity sets for plurisubharmonic functions and related problems, Proc. London Math. Soc. (3) 36 (1978), 310-336.

3. J. E. Fornaess and N. Sibony, Plurisubharmonic functions on ring domains, Complex Analysis (S. G. Krantz, Ed.), Lecture Notes in Math., vol. 1268, Springer, Berlin and New York.

4. P. Griffith, Two theorems on extension of holomorphic mappings, Invent. Math. 14 (1971), 27-62.

Department of MAthematics, University of UMEA, 90197 UMEA, SWEDEN 